\title{
UNIQUENESS THEOREMS FOR A GENERAL CLASS OF FUNCTIONAL EQUATIONS
}

\author{
C. T. NG
}

(Received 19 February 1969)

Communicated by J. B. Miller

In a previous paper [1] J. Aczél has shown the following $\langle A, B\rangle$

THEOREM 1. If in the (closed, half-closed or open, finite or infinite) interval

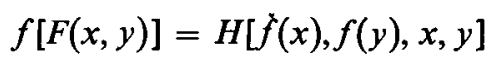

and there $f, F$ are continuous, $F$ intern (the value $F(x, y)$ lies strictly between $x$ and $y)$ and $u \rightarrow H(u, v, x, y)$ or $v \rightarrow H(u, v, x, y)$ are injective (i.e.

$$
H\left(u_{1}, v, x, y\right)=H\left(u_{2}, v, x, y\right) \Rightarrow u_{1}=u_{2}
$$

or

$$
\left.H\left(u, v_{1}, x, y\right)=H\left(u, v_{2}, x, y\right) \Rightarrow v_{1}=v_{2}\right),
$$

then the functional equation (*) with the initial conditions

$$
f(a)=c, f(b)=d \quad(a, b \in\langle A, B\rangle)
$$

has at most one solution.

The above result has been established for functions with real variables. In the sequel we extend the notion of internness to vector spaces and derive results in topological vector spaces.

DEFINITIONS, NOTATIONS AND PRELIMINARIES: For two distinct points $x$ and $y$ of a vector space (v.s.) $E$ over the real field $\mathbf{R}$, we denote the line joining $x$ and $y$ by

$$
L\langle x, y\rangle=\{y+t(x-y): t \in \mathbf{R}\}
$$

and the (open) line segment joining $x$ and $y$ by

$$
L(x, y)=\{y+t(x-y): t \in(0,1)\} .
$$

A mapping $F$ defined on some subset $S$ of $E \times E$ into $E$ is said to be intern if

$$
F(x, y) \in L(x, y)
$$

whenever $(x, y) \in S$ with $x \neq y$.

Given a vector space $E$ over the real field $\mathbb{R}$ and a topology $\mathfrak{T}$ on $E$, the pair 
$(E, \mathfrak{I})$ is called a topological vector space (t.v.s.) over $\mathbb{R}$ if these two axioms are satisfied:

$$
\begin{aligned}
& (L T)_{1}(x, y) \rightarrow x+y \text { is continuous on } E \times E \text { into } E, \\
& (L T)_{2}(t, x) \rightarrow t x \text { is continuous on } \mathrm{R} \times E \text { into } E .
\end{aligned}
$$

The field $\mathbf{R}$ is always considered to be endowed with its usual absolute value, under which it is a non-discrete valuated field. In addition it is always considered under its usual order ([6], page 11-12). Every t.v.s. $E$ considered here is over the real field $\mathbf{R}$ if the field is not mentioned.

If $E$ is a Hausdorff topological vector space over $\mathbf{R}$ and $\mathfrak{I}$ is the relative topology on $L\langle x, y\rangle$, the injective mapping $\Phi_{x}^{y}$ of $L\langle x, y\rangle$ onto $\mathbb{R}$ defined by

$$
\Phi_{x}^{y}(y+t(x-y))=t \quad \text { for all } t \in \mathbf{R}
$$

is a homeomorphism ([6], page 21).

If $E$ and $N$ are topological spaces with $N$ Hausdorff and $f_{1}, f_{2}: E \rightarrow N$ are continuous mappings, then the set

is closed in $E$.

$$
S=\left\{x: x \in E, f_{1}(x)=f_{2}(x)\right\}
$$

THEOREM 2. Let $E_{1}$ be a closed subset of $a$ Hausdorff t.v.s. $E$ and let $F: E_{1} \times E_{1} \rightarrow E_{1}$ be intern, continuous in both variables. Let $N$ be a set and $f_{1}, f_{2}: E_{1} \rightarrow N$ be mappings satisfying the functional equation

$$
f[F(x, y)]=H[f(x), f(y), x, y]
$$

where the mapping $H: N \times N \times E_{1} \times E_{1} \rightarrow N$ is injective either in its first variable or in its second variable. If $f_{1}$ and $f_{2}$ are identical on some $E_{1}$-neighbourhood $V$ of a point $a \in E_{1}$, then $f_{1}$ and $f_{2}$ are identical on the entire domain $E_{1}$.

Proor. Without loss of generality we may suppose $H$ is injective in its first variable. Let $x_{0} \in E_{1} \backslash\{a\}$ be given. Define

and

$$
F^{0}(x, a)=x
$$

$$
F^{n+1}(x, a)=F\left(F^{n}(x, a), a\right)
$$

recursively for $x \in E_{1}$ and $n \in \omega=\{0,1,2, \cdots\}$. It follows from the internness of $F$ that $\left\langle\Phi_{x_{0}}^{a}\left(F^{n}\left(x_{0}, a\right)\right)\right\rangle_{n \in \omega}$ is a decreasing sequence of positive real numbers and therefore converges to a definite limit, say

$$
\lim _{n \rightarrow \infty} \Phi_{x_{0}}^{a}\left(F^{n}\left(x_{0}, a\right)\right)=\alpha \geqq 0 .
$$

We now claim that the sequence $\left\langle F^{n}\left(x_{0}, a\right)\right\rangle_{n \in \omega}$ in $L\left\langle x_{0}, a\right\rangle$ converges to $a$. For $\Phi_{x_{0}}^{a}$ is a homeomorphism so that 


$$
\lim _{n \rightarrow \infty} F^{n}\left(x_{0}, a\right)=\Phi_{x_{0}}^{a-1}(\alpha) \in \bar{E}_{1}=E_{1}
$$

and the fact that $F$ is continuous in its first variable yields

$$
\begin{aligned}
F\left(\Phi_{x_{0}}^{a-1}(\alpha), a\right)= & F\left(\lim _{n \rightarrow \infty} F^{n}\left(x_{0}, a\right), a\right) \\
& =\lim _{n \rightarrow \infty} F\left(F^{n}\left(x_{0}, a\right), a\right) \\
& =\lim _{n \rightarrow \infty} F^{n+1}\left(x_{0}, a\right) \\
& =\Phi_{x_{0}}^{a-1}(\alpha) .
\end{aligned}
$$

In view of the internness of $F$, we must have $\Phi_{x_{0}}^{a-1}(\alpha)=a$ and convergence of $\left\langle F^{n}\left(x_{0}, a\right)\right\rangle_{n \in \omega}$ to its limit $a$ follows. Since $V$ is an $E_{1}$-neighbourhood of $a$, there exists $N \in \omega \backslash\{0\}$ such that

and consequently

$$
F^{N}\left(x_{0}, a\right) \in V
$$

By (*) we have

$$
f_{1}\left(F^{N}\left(x_{0}, a\right)\right)=f_{2}\left(F^{N}\left(x_{0}, a\right)\right)
$$

$$
\begin{aligned}
H\left[f_{1}\right. & \left.\left(F^{N-1}\left(x_{0}, a\right)\right), f_{1}(a), F^{N-1}\left(x_{0}, a\right), a\right] \\
& =f_{1}\left(F^{N}\left(x_{0}, a\right)\right) \\
& =f_{2}\left(F^{N}\left(x_{0}, a\right)\right) \\
& =H\left[f_{2}\left(F^{N-1}\left(x_{0}, a\right)\right), f_{2}(a), F^{N-1}\left(x_{0}, a\right), a\right]
\end{aligned}
$$

so that

$$
f_{1}\left(F^{N-1}\left(x_{0}, a\right)\right)=f_{2}\left(F^{N-1}\left(x_{0}, a\right)\right)
$$

follows from the injectivity assumption on $H$ in its first variable. Proceeding recursively we obtain

or simply

$$
f_{1}\left(F^{0}\left(x_{0}, a\right)\right)=f_{2}\left(F^{0}\left(x_{0}, a\right)\right)
$$

$$
f_{1}\left(x_{0}\right)=f_{2}\left(x_{0}\right)
$$

The point $x_{0} \in E_{1} \backslash\{a\}$ being arbitrary, $f_{1}$ and $f_{2}$ are identical on $E_{1}$.

THEOREM 3. Let $F: E_{1} \times E_{1} \rightarrow E_{1}$ be an intern function defined on a closed subset $E_{1}$ of a Hausdorff t.v.s. $E$ over $\mathbf{R}$, and let $N$ be a Hausdorff space. Suppose $f_{1}, f_{2}: E_{1} \rightarrow N$ are continuous mappings satisfying

$$
f[F(x, y)]=H[f(x), f(y), x, y]
$$

where $H$ is a mapping from $N \times N \times E_{1} \times E_{1}$ into $N$. Then the set

is closed and convex.

$$
S=\left\{x: x \in E_{1}, f_{1}(x)=f_{2}(x)\right\}
$$


Proof. Since $E_{1}$ is closed in $E$ and $S$ is closed in $E_{1}, S$ is also closed in $E$. For any two distinct points $x$ and $y$ of $S$, consider the line $L\langle x, y\rangle$ and its relative topology $\mathfrak{I}$. The map $\Phi_{x}^{y}: L\langle x, y\rangle \rightarrow \mathbf{R}$ is a homeomorphism. In view of this homeomorphism $L(x, y) \backslash S$ is open and can be represented as a countable union of disjoint open components (or equivalently, open intervals, open segments). Suppose, if possible, that $L(x, y) \backslash S$ is non-empty and there exists an open component of it, say $L\left(x_{1}, y_{1}\right)$. We must have $x_{1}, y_{1} \in S$ and $L\left(x_{1}, y_{1}\right) \cap S=\emptyset$. However by $(*)$

$$
\begin{aligned}
f_{1}\left(F\left(x_{1}, y_{1}\right)\right) & =H\left[f_{1}\left(x_{1}\right), f_{1}\left(y_{1}\right), x_{1}, y_{1}\right] \\
& =H\left[f_{2}\left(x_{1}\right), f_{2}\left(y_{1}\right), x_{1}, y_{1}\right] \\
& =f_{2}\left[F\left(x_{1}, y_{1}\right)\right]
\end{aligned}
$$

we have $F\left(x_{1}, y_{1}\right) \in S$. Also by the interness of $F, F\left(x_{1}, y_{1}\right) \in L\left(x_{1}, y_{1}\right)$. Now $F\left(x_{1}, y_{1}\right) \in L\left(x_{1}, y_{1}\right) \cap S$ leads to a contradiction. So $L(x, y) \backslash S=\emptyset$ and $L(x, y) \subseteq S$. The points $x, y$ being arbitrary, $S$ is convex.

We are now able to state our final result which is an immediate consequence of the previous two theorems.

THEOREM 4. Let $E_{1}$ be a closed subset of a Hausdorff t.v.s. E, and let $F: E_{1} \times E_{1} \rightarrow E_{1}$ be intern, continuous in both variables. Let $N$ be a Hausdorff space. Suppose $f_{1}, f_{2}: E_{1} \rightarrow N$ are continuous mappings satisfying the functional equation

$$
f[F(x, y)]=H[f(x), f(y), x, y]
$$

where the mapping $H: N \times N \times E_{1} \times E_{1} \rightarrow N$ is either

injective in its first variable

or$$
\text { i.e. } H\left(u_{1}, v, x, y\right)=H\left(u_{2}, v, x, y\right) \Rightarrow u_{1}=u_{2}
$$

If $f_{1}$ and $f_{2}$ are identical on some subset $A$ of $E_{1}$ whose closed convex hull $\overline{\Gamma A}$ has non-empty interior in $E_{1}$ (interior taken in $E_{1}$ ), then $f_{1}$ and $f_{2}$ are identical on the entire $E_{1}$.

Corollary. If in Theorem $4, E$ is locally convex Hausdorff of dimension $n$ and $A=\left\{a_{i}: i=1,2, \cdots, n+1\right\}$ is such that $\left\{a_{i}-a_{1}: i=2,3, \cdots, n+1\right\}$ is linearly independent, then there exists at most one continuous solution of $(*)$ satisfying the $n+1$ initial conditions

$$
f\left(a_{i}\right)=b_{i} \quad i=1,2, \cdots, n+1 .
$$

Remarks. In case $n=1$, this gives Theorem 1 for closed $\langle A, B\rangle$. However the assumption that $\langle A, B\rangle$ is closed may be omitted as any interval $\langle A, B\rangle$ 
with $a, b \in\langle A, B\rangle$ can be represented as a union of closed intervals containing $a$ and $b$. For Fréchet spaces, $A$ may be chosen as a subset whose closed convex hull $\overline{\Gamma A}$, after a translation, contains a barrel.

Theorems 2, 3 and 4 are still valid if we do not assume $E$ to be Hausdorff. In this case the map $\Phi_{x}^{y}$ may fail to be a homeomorphism, and this happens only when $\mathfrak{I}$ is indiscrete (on $L\langle x, y\rangle$ ). Extra discussions lead to the same conclusions.

\section{References}

[1] J. Aczél, 'Ein Eindeutigkeitssatz in der Theorie der Funktionalgleichungen und einige ihrer Anwendungen', Acta Math. Acad. Sci. Hung. 15 (1964), 355-362.

[2] J. Aczél and M. Hosszú, 'Further Uniqueness Theorems for Functional Equations', Acta Math. Acad. Sci. Hung. 16 (1965), 51-55.

[3] J. Aczél, 'On Applications and Theory of Functional Equations' (Birkhäuser, Basel, 1969, $22-25)$.

[4] Hans-Jürgen Albrand, 'Eindeutigkeitssätze für Funktionalgleichungen', 1968, Thesis.

[5] T. D. Howroyd, 'Some Uniqueness Theorems for Functional Equations', J. Austral. Math. Soc. 9 (1969), 176-179.

[6] H. H. Schaefer, 'Topological Vector Spaces', (Macmillan, 1966).

University of Waterloo

Waterloo, Ontario, Canada 